\title{
Provider behavior change approaches to improve family planning services in the Ouagadougou partnership countries: A landscaping review
}

\author{
Kathryn Spielman \\ Population Council \\ Elizabeth Tobey \\ Population Council \\ Martha Silva \\ Leanne Dougherty \\ Population Council
}

Follow this and additional works at: https://knowledgecommons.popcouncil.org/departments_sbsr-rh

Part of the Health Communication Commons, and the Public Health Commons

How does access to this work benefit you? Let us know!

\section{Recommended Citation}

Spielman, Kathryn, Elizabeth Tobey, Martha Silva, and Leanne Dougherty. 2020. "Provider behavior change approaches to improve family planning services in the Ouagadougou partnership countries: A landscaping review," Breakthrough RESEARCH Final Report. Washington, DC: Population Council. 


\section{LANDSCAPE REVIEW}

Provider Behavior Change Approaches to Improve Family Planning Services in the Ouagadougou Partnership Countries: A Landscaping Review

FEBRUARY 2020

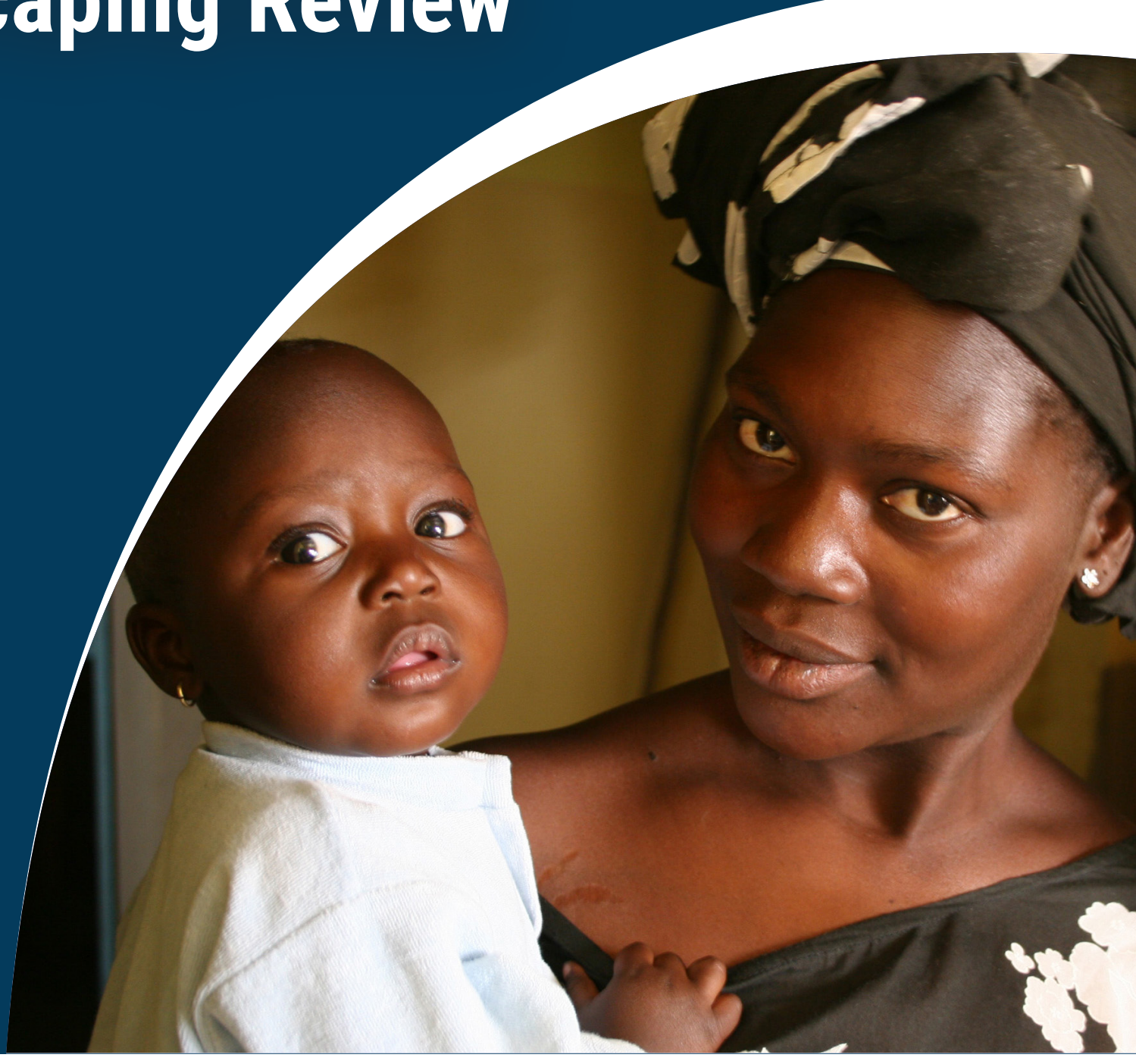




\section{Acknowledgments}

We acknowledge the teams from Pathfinder International, YLabs, and Breakthrough ACTION for their insights, particularly Theo Gibbs and Rebecca Hope of YLabs and Jessica Vandermark of Camber Collective for their contributions to the case studies and their overall review. We acknowledge Paul C. Hewett and Sanyukta Mathur of Population Council for their technical guidance and review. We acknowledge Mariko Hewer and Sherry Hutchinson of Population Council, who provided editorial and design support. We acknowledge Hope Hempstone, Lindsay Swisher, Joan Kraft, and Jaqueline Devine with the United States Agency for International Development (USAID), who provided valuable feedback during the development of this report.

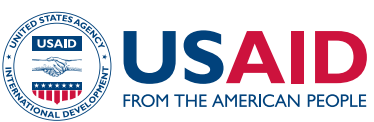

Breakthrough RESEARCH is made possible by the generous support of the American people through the United States Agency for International Development (USAID) under the terms of cooperative agreement no. AID-OAA-A-17-00018. The contents of this document are the sole responsibility of the Breakthrough RESEARCH and Population Council and do not necessarily reflect the views of USAID or the United States Government.

\section{Tulane University}

As stewards of the first school of public health in the United States, the Tulane University School of Public Health and Tropical Medicine cultivates independent thinkers, innovative leaders, fierce advocates, and accomplished scholars.

From the neighborhoods of New Orleans to communities worldwide, we conduct research and collaborate with our partners to ensure that all of humanity has an equitable opportunity to be healthy and pursue optimal well-being.

We train the problem solvers. Find us on the Front Lines.
(C2020 The Population Council. All rights reserved.

Cover photo by (C2006 Richard Nyberg, Courtesy of Photoshare

\section{Suggested Citation}

Spielman, Kathryn, Elizabeth Tobey, Martha Silva, and Leanne Dougherty. 2020. "Provider behavior change approaches to improve family planning services in the Ouagadougou Partnership Countries: a landscaping review," Breakthrough RESEARCH Final Report. Washington DC: Population Council.

\section{Contact}

4301 Connecticut Avenue NW, Suite 280 | Washington, DC 20008 +12022379400 | BreakthroughResearch@popcouncil.org

breakthroughactionandresearch.org

\section{POPULATION COUNCIL \\ Ideas. Evidence. Impact.}

The Population Council confronts critical health and development issuesfrom stopping the spread of HIV to improving reproductive health and ensuring that young people lead full and productive lives. Through biomedical, social science and public health research in about 50 countries, the Council works with our partners to deliver solutions that lead to more effective policies, programs, and technologies to improve lives worldwide. Established in 1952 and headquartered in New York, the Council is a nongovernmental, nonprofit organization with an international board of trustees.

\section{Breakthrough RESEARCH FOR SOCIAL \& BEHAVIOR CHANGE}

Breakthrough RESEARCH catalyzes social and behavior change (SBC) by conducting state-of-the-art research and evaluation and promoting evidence-based solutions to improve health and development programs around the world. Breakthrough RESEARCH is a consortium led by the Population Council in partnership with Avenir Health, ideas42, Institute for Reproductive Health at Georgetown University, Population Reference Bureau, and Tulane University. 


\section{Provider Behavior Change Approaches to Improve Family Planning Services in the Ouagadougou Partnership Countries: A Landscaping Review}

Kathryn Spielman ${ }^{1} \quad$ Martha Silva ${ }^{2}$

Elizabeth Tobey ${ }^{1} \quad$ Leanne Dougherty ${ }^{1}$

${ }^{1}$ Population Council

${ }^{2}$ Tulane University 


\section{List of Acronyms}

CHW Community Health Worker

DEC Development Experience Clearinghouse

E2A Evidence to Action

FP Family Planning

FWA Francophone West Africa

HC3 Health Communication Capacity Collaborative

HIV Human Immunodeficiency Virus

IUD Intrauterine Device

LAPMs Long-Acting and Permanent Methods

LMIC Low- and Middle-income Countries

OP Ouagadougou Partnership

PAC Post-Abortion Care

PBC Provider Behavior Change

RBF Results-based Financing

RCT Randomized Controlled Trial

$\mathrm{RH} \quad$ Reproductive Health

SBC Social and Behavior Change

URC University Research Co.

WHO World Health Organization 


\section{Table of Contents}

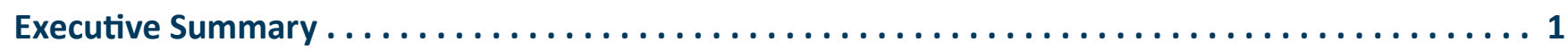

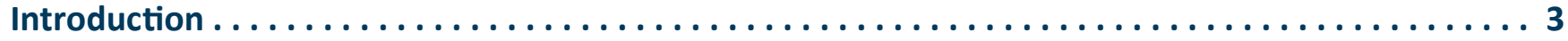

Objective....................................................... 4

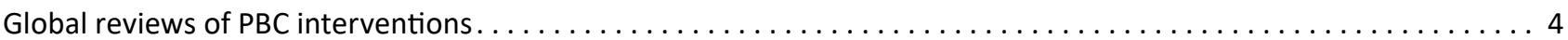

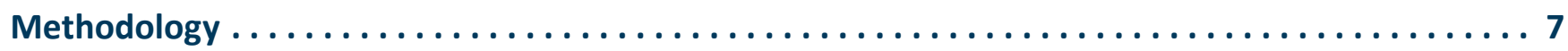

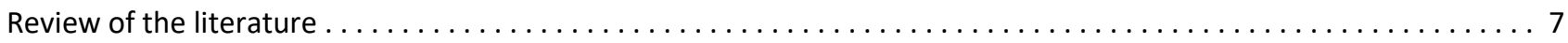

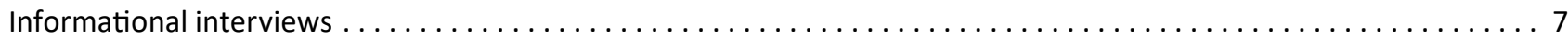

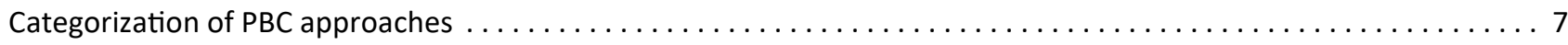

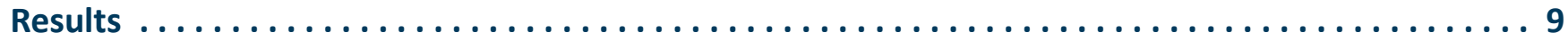

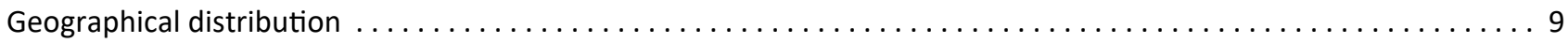

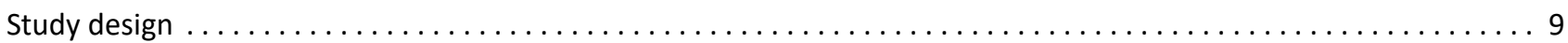

Barriers addressed $\ldots \ldots \ldots \ldots \ldots \ldots \ldots \ldots \ldots \ldots \ldots \ldots \ldots \ldots \ldots \ldots \ldots \ldots \ldots \ldots$

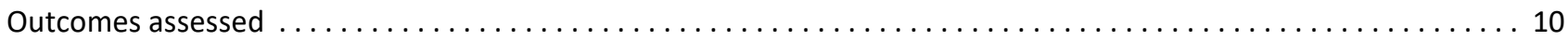

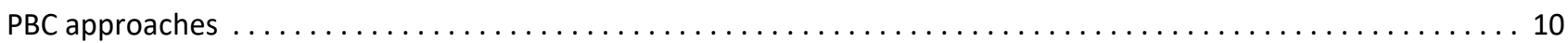

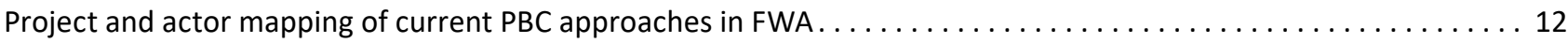

Case Study 1: Quality Assurance Project in Niger-Camber Collective and Animas Sutura . . . . . . . . . . . . 12

Case Study 2: Beyond Bias in Burkina Faso-YLabs, Camber Collective, and Pathfinder International . . . . . . . . . 14

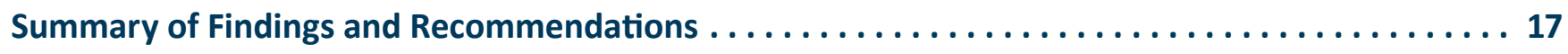

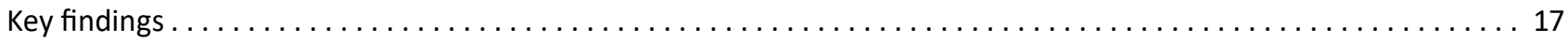

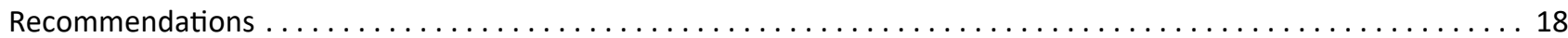

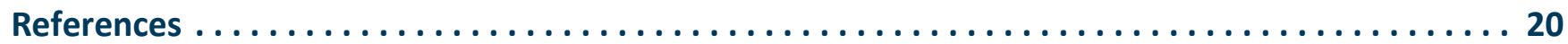

Appendices can be found online at breakthroughactionandresearch.org/wp-content/ uploads/2020/03/PBC-Landscaping-FP-OP-Supp.pdf 


\section{Executive Summary}

\section{This landscaping review summarizes the available evidence on the effectiveness of pro- vider behavior change (PBC) interventions for improving family planning (FP) services in the Ouagadougou Partnership (OP) countries and presents current or recent PBC interventions in OP countries that have not been formally evaluated. The findings are summarized along with reflections about possible research and programmatic investments to address challenges and opportunities identified.}

Effective client-provider interactions are essential to consistent demand and uptake of family planning services. The quality of a client-provider interaction can be influenced by a provider's knowledge, attitudes, biases, and expectations, as well as structural factors such as method availability or privacy of counseling space. The United States Agency for International Development's (USAID) Breakthrough RESEARCH project conducted a landscaping review to explore PBC approaches for improving FP services in the OP countries. For purposes of this review, we define $\mathrm{PBC}$ interventions to include interventions that go beyond clinical training and support (e.g., technical job aids) and seek to positively influence provider behavior to improve the quality of services, enhance client experiences, increase demand for services, and increase uptake of commodities or adoption of healthier behaviors.

The landscaping review focused on the OP countries and included a literature search and informational interviews with project staff who have contributed to PBC implementation activities or evaluations in Francophone West Africa (FWA). We searched Pubmed (from 2000 to present) for peer-reviewed literature as well as the USAID Development Experience Clearinghouse, conference websites including the International Conference on Family Planning and the Sommet Francophone pour le Changement Social et de Comportement, and PBC implementing partner websites for grey literature. Because the term PBC is not broadly used, we focused the search on strategies used to improve health care provider practices as identified in a recent global review. We created matrices capturing key details on geographic location, key outcomes and approach to facilitate comparisons across studies. We complemented this literature search with informational interviews. We conducted interviews from August through September 2019 and interviewed representatives from organizations working on PBC. We requested information on the nature, scope and, where possible, the impact of the PBC interventions.

Studies were limited in geographic scope. Most evaluations focused on interventions in four countries: Senegal, Guinea, Mali, and Niger, the four OP countries with the lowest unmet need for FP. There were gaps in the PBC approaches used and barriers to provider behavior addressed: only one study explicitly targeted provider attitudes/biases as part of a provider training. Most studies did not assess the impact of the PBC interventions on provider-level outcomes, such as provider knowledge or attitudes. Studies more often assessed client FP uptake, a more distal outcome, and most did so using routinely collected monitoring data on client outcomes without design or analytical techniques to allow causal inference. There is a need to assess provider-level outcomes to validate the theory of change and ensure that the expected outcome among providers was achieved. Although the studies generally found positive effects on outcomes, the sustainability of improvements was not assessed, and only one study collected data on cost. Despite gaps in the documented evidence, there are many ongoing PBC studies in OP countries that may address these questions in the next few years.

Previous studies have found that provider knowledge, attitudes, and biases influence family planning service utilization. PBC interventions offer a promising approach to address these barriers to service utilization. However, there is limited evidence on effective approaches in OP countries. Ongoing evaluations will be completed in the next five years and will inform future programs aimed at improving client-provider interactions. Findings from the landscaping study suggest that future programs should consider focusing on documenting interventions' underlying theories of change, as well as early lessons learned from prototyping and formative work, in order to strengthen the evidence base. In addition, there is a need 
for rigorous evidence on what works in PBC, and, when possible, evaluations should employ randomized designs, comparison groups, and/or triangulation with routine or program monitoring data. Lastly, the scope of evaluations should be expanded to assess interventions focused on provider attitudes and provider bias, provider-level outcomes, PBC approaches including social accountability and incentives, and impacts of interventions on vulnerable populations, including youth and nulliparous women in FWA. 


\section{Introduction}

Over the past several decades, USAID investments to promote the use of family planning (FP) in Francophone West Africa (FWA) have focused on procurement and supply of commodities, with less investment in social and behavior change (SBC) directed toward increasing demand, facilitating decision-making around use of contraception, and enhancing the enabling environment for positive health behaviors. ${ }^{1}$ This is the case despite the relatively low demand for FP in the region: $\mathbf{4 0 . 2 \%}$ in Western Africa compared with $\mathbf{5 7 . 7 \%}$ globally. ${ }^{b, 2}$ Implementing partners working to promote FP use in FWA have centered their activities on either improving service delivery or SBC for demand creation, with little consideration of the potential areas of intersection. The provider-client interaction is an area of intersection that has been considered an element of the quality of care ${ }^{3,4}$ but is usually not considered a potential area for SBC interventions. However, in the field of $\mathrm{SBC}$, addressing provider behavior is an increasingly important component of SBC, as it is perceived as influencing decision-making around FP, the demand for FP, and the uptake of contraception. ${ }^{5}$ Further, it is increasingly recognized that provider behavior is influenced by factors beyond their clinical training that fall within the purview of SBC, including norms, attitudes, cognitive biases, and motivations. ${ }^{6}$

Despite the relative lack of attention to provider behavior in SBC interventions, recent projects in FWA have investigated barriers to effective provider behavior change (PBC). A 2016 review by the Health Communication Capacity Collaborative (HC3) identified three categories of barriers to service provision:?

- Knowledge and competency barriers: Providers do not know how to perform assigned tasks.

- Structural and contextual barriers: Providers are not able to perform assigned tasks.

- Attitudinal barriers: Providers are not willing to perform assigned tasks.

Each of these barriers exists for FP providers within FWA. Qualitative evidence from Côte d'Ivoire reveals that providers may promote the methods that they feel the most comfortable explaining and know the most about. ${ }^{8}$ From qualitative research, structural and contextual barriers cited in the region include frequent stockouts and a lack of space dedicated to FP counseling. ${ }^{8}$ Providers report that they often resort to counseling on the FP methods that are in stock at the moment rather than informing clients about all FP methods. Attitudinal barriers also exist for providers in the region. In urban Senegal, Sidze et al. described provider-imposed restrictions on FP methods based on perceived client characteristics. ${ }^{9}$ For example, providers-in particular, male providers - set self-imposed age minimums for the pill and injectables, while older providers set self-imposed restrictions based on marital status. In Burkina Faso, as well as in Tanzania and Pakistan, the Beyond Bias project has found provider-imposed bias based on parity, in which providers deny services to women with no or few children. ${ }^{10}$

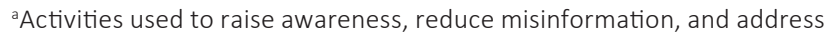
the barriers that prevent individuals, families, and communities from practicing lifesaving behaviors to improve health outcomes.

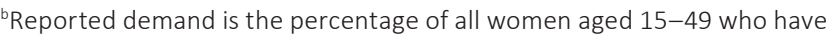
a demand for FP, including those with met and unmet needs. 


\section{Objective}

The purpose of this landscaping is to summarize the available evidence on the effectiveness of PBC interventions for improving FP services in the Ouagadougou Partnership (OP) countries ${ }^{c}$. For purposes of this review, we define $\mathrm{PBC}$ interventions to include interventions that go beyond clinical training and support (e.g., technical job aids) and seek to positively influence provider behavior to improve the quality of services, enhance client experiences, increase demand for services, and increase uptake of commodities or adoption of healthier behaviors. ${ }^{11}$ The document synthesizes both published and grey literature, with additional insights gathered from informational interviews about projects that are testing PBC approaches. It also presents current or recent PBC interventions in OP countries that have not been formally evaluated. The findings are summarized along with reflections about possible research and programmatic investments to address challenges and opportunities identified.

\section{Global reviews of PBC interventions}

This landscaping builds upon two global reviews of the effectiveness of PBC interventions.

A 2018 global review by Alexander Rowe and colleagues synthesized the evidence on the effectiveness of strategies to improve healthcare provider practices across multiple health areas in low- and middle-income countries (LMICS). ${ }^{12}$ Rowe and colleagues searched 110 electronic databases for both published and unpublished studies from the 1960 s to 2016 and selected 670 reports from 337 studies of 118 strategies for their review. This review synthesized evidence from 64 countries, although little literature based in FWA was found.

The reviewed studies included provider-level interventions, such as supervision and training, as well as

\footnotetext{
'The partnership was launched in Ouagadougou, Burkina Faso, in February 2011 at the Regional Conference on Population, Development and Family Planning held by the nine governments of Francophone West African countries (Benin, Burkina Faso, Côte d'Ivoire, Guinea, Mali, Mauritania, Niger, Senegal, and Togo) and their technical partners. It seeks to mobilize financial resources to accelerate progress in the use of FP services in the Partnership countries (https://parternariatouaga.org/en/).
}

health-systems-level interventions to improve infrastructure, health financing, and management. Rowe et al. denoted 13 different programmatic strategies: 1) enhanced community support, 2) patient support (including patient health education and home visits), 3) strengthened infrastructure, 4) provider financial incentives, 5) health system financing and incentives, 6) heath system regulation and governance, 7) group problem-solving with providers, 8) improved supervision, 9) management strategies and techniques, 10) highintensity training, 11) low-intensity training, 12) printed information (including job aids), and 13) information and communication technology.

Rowe et al. found varied effectiveness of these different strategies to improve targeted provider practice outcomes across time (i.e., patient assessment, diagnosis, and treatment). For facility-based providers, interventions that included only technology-based strategies or printed information for providers had near zero effects on performance. Interventions addressing provider training or supervision alone had moderate effects (10.3-15.9 percentage points) on practice outcomes, but when combined had larger effects on provider performance (18.0-18.8 percentage points). Group problem-solving showed large improvements for provider performance (28.0-37.5 percentage points). Many interventions used multifaceted strategies and had large effects on provider performance, though these were not always more effective than simpler strategies. For community health workers (CHWs), community support approaches, including community health education or social marketing of health services combined with training, had large effects on performance (8.2-125.0 percentage points). Studies were only included if they used strategy versus control group comparisons. Unfortunately, most strategies had low-quality evidence, and differences in context and methodologies made comparisons challenging.

As the scope of the Rowe et al. review was limited to interventions that address provider performance, these approaches primarily sought to address knowledge and competency barriers or structural and contextual barriers to service provision. Other intervention approaches, such as incentives or managerial techniques, may have also addressed attitudinal barriers by increasing the 
internal motivation of providers. However, Rowe et al. did not include provider bias, which is a notable attitudinal barrier within the field of FP, in their search, explicitly through search terms or by separating interventions that could address provider bias in their screening of the studies. Furthermore, among 337 reviewed studies, only 15 were from OP countries. Only seven of the nine OP countries had any evidence: Rowe and colleagues did not find any PBC studies from Mauritania or Guinea, and only reviewed one each from Togo and Senegal. Most (12) of the 15 studies examined provider performance for maternal and child health care, and two of the remaining three interventions related to HIV/AIDS and malaria care, respectively. Only one evaluated a program aiming to improve the performance of FP providers, and it was published in 1994.

Julie Solo and Mario Festin recently published a review of provider bias in FP services to understand its definition, breadth, and impact, as well as approaches that successfully address provider bias. ${ }^{13}$ Solo and Festin describe a conceptual framework produced by the World Health Organization (WHO) (see figure 1) in which a provider's core beliefs, local values and norms, empathy for clients, and socialization affect a provider's attitudes, which can also be affected by interventions such as supervision, training, values clarification, or incentives. Provider attitudes may manifest through the provider actions, which can also be influenced by regulations, policies, infrastructure, supplies, and workload. Thus, provider biases are only a barrier to care if the resulting actions from these biases negatively affect FP service provision, the ability of clients to make informed choices, and/or clients' confidence and ability to act on choices made. Providers may hold both implicit biases (unintentional and unconscious) influenced by social factors and explicit biases (conscious and intentional); both may negatively affect quality of care and therefore both should be examined and addressed. Furthermore, although Solo and Festin reviewed evidence that identified provider-imposed restrictions based on age and marital status in Senegal and Burkina Faso, their review did not find any evidence of effectiveness of PBC interventions in the FWA region and found limited evidence globally.

Reviewing approaches that address bias, Solo and Festin cite that simply giving providers information about methods and their clinical guidelines for use typically does not reduce bias. A number of studies examined found mixed results on the effectiveness of training on clinical standards for contraceptive method use, often finding that providers continued to impose their own standards for appropriate contraceptive methods after training. Solo and Festin suggest a number of principles to consider when designing interventions to reduce bias, though there is little evidence provided on the effectiveness

\section{FIGURE 1 CONCEPTUAL FRAMEWORK OF PROVIDERS' INFLUENCE ON CLIENT UTILIZATION OF SEXUAL AND REPRODUCTIVE HEALTH SERVICES ${ }^{14}$}

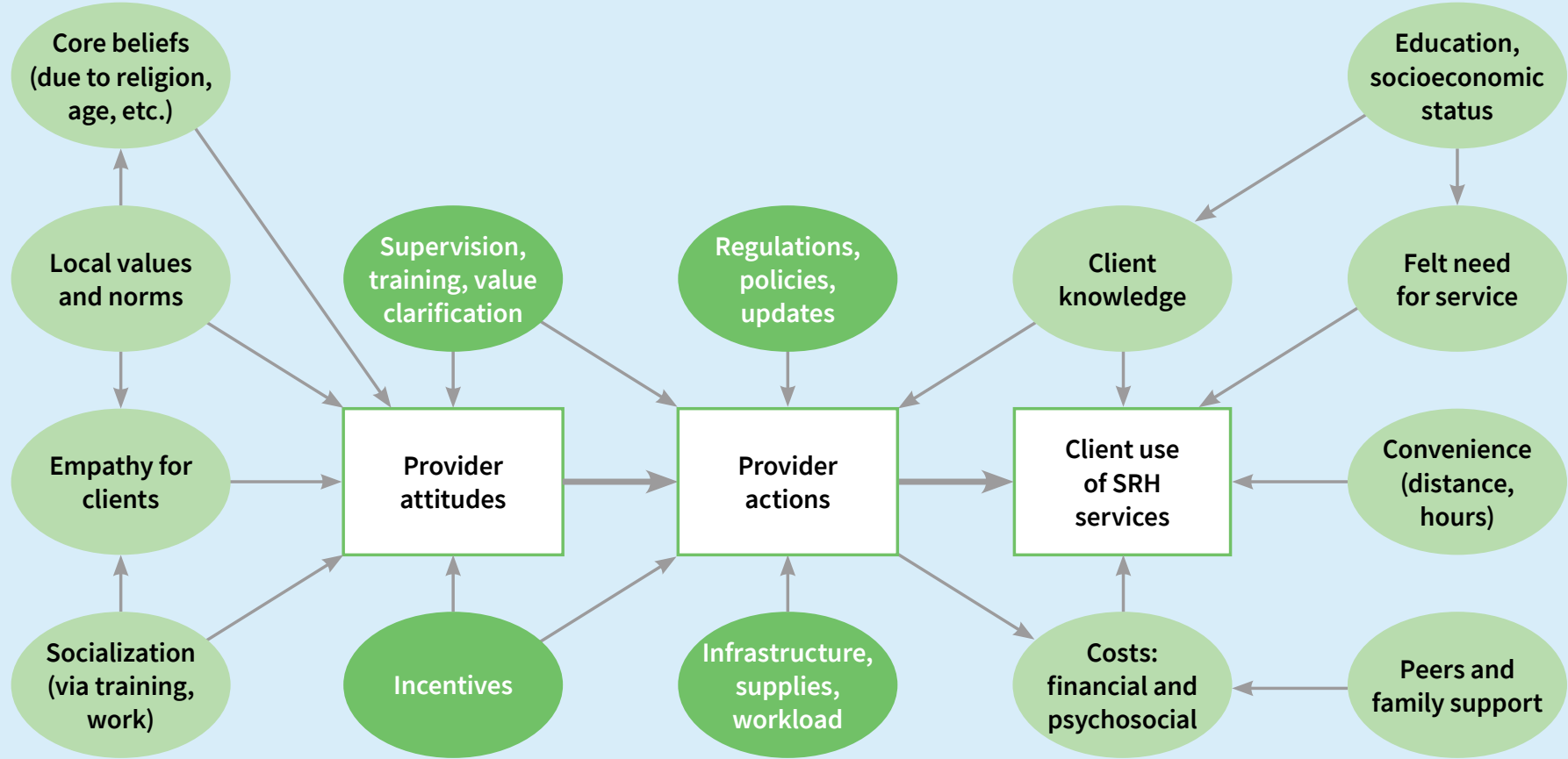

Abbreviation: $\mathrm{SRH}=$ sexual and reproductive health 
of these principles. They include: 1) avoiding blaming providers for bias and instead employing a supportive approach, 2) training a small number of early adopters or dedicated providers who can act as role models and mentor colleagues, 3) using more comprehensive SBC approaches, and 4) being clear and proactive about nondiscrimination when implementing new guidelines for care and training providers.

Given the lack of synthesized evidence on PBC interventions for FP in FWA, this landscaping aims to describe the various approaches that have been used in evaluated interventions to address provider behavior in FWA for $F P$, synthesize the evidence on the effectiveness of these approaches, and summarize gaps and opportunities to advance the evidence base for PBC in this region. 


\section{Methodology}

This landscaping review was conducted in two parts and is further described below. First, a review of the literature was conducted using publicly available databases. Next, we complemented this search strategy with informational interviews with project staff who have contributed to PBC implementation activities or evaluations in FWA.

\section{Review of the literature}

We searched PubMed for peer-reviewed articles. The search terms applied in the PubMed database included the population of interest (providers OR community health worker) AND evaluation studies (evaluation OR effective*) ${ }^{d}$ AND reproductive health (family planning OR contraception*) AND provider outcomes of interest (quality OR provider behavior change OR attitudes) AND with a focus on the OP countries (Benin OR Burkina Faso OR Côte d'Ivoire OR Guinea OR Mali OR Mauritania OR Niger OR Senegal OR Togo). We searched the USAID Development Experience Clearinghouse (DEC), conference websites including the International Conference on Family Planning and the Sommet Francophone pour le Changement Social et de Comportement, and PBC implementing partner websites for grey literature (Appendix 1). ' Using the DEC's advanced search tool, technical documents from the nine countries were sought using the following key words: provider, community health worker, quality, attitudes, and behavior. The International Conference on Family Planning and the Sommet Francophone pour le Changement Social et de Comportement programs were searched using the terms "provider" and "worker" and "prestataires," "agent," and "travailleur." As there were fewer documents expected to be available in grey literature, we broadened the search, dropping some of the previously used phrases such as "evaluation" or "effective." All results were examined to ensure that they included evaluations of a $\mathrm{PBC}$ intervention.

\footnotetext{
${ }^{\mathrm{d}}$ Asterisk denotes that PubMed searches for any word with this root (i.e.,
} contraceptive, contraception).

eAvailable at breakthroughactionandresearch.org/wp-content/ uploads/2020/03/PBC-Landscaping-FP-OP-Supp.pdf

\section{Additional inclusion criteria}

- Were written in English or French

- Were published in 2000 or later

- Included at least one PBC approach (see Table 1)

- Included at least one FP/reproductive health (RH) outcome

- Were located, at least in part, in one of the nine OP countries

- Included an evaluation component (for the evaluation synthesis section only)

\section{Informational interviews}

Three interviews were conducted with project staff from recent and ongoing PBC projects to understand the types of interventions being implemented and to learn about any undocumented findings or lessons learned. Any ongoing projects in FWA mentioned by respondents were added to the database of current or recent projects. Two ongoing projects that had preliminary results with compelling evidence were included as case studies.

The first respondent was identified by an internal source, and the subsequent informants were suggested by the first respondent during the first interview. The organizations represented were Camber Collective, Pathfinder International, and YLabs. Interviews were conducted by phone in August and September 2019. Participants were informed about potential inclusion of the contents of the interview in the landscaping document, and all participants consented. Participants also reviewed and provided feedback on the drafted landscaping document.

\section{Categorization of PBC approaches}

We derived categories of PBC approaches under which the approaches used in the included studies could be grouped from the conceptual framework developed by WHO and presented in Solo et al. 2019. Subcategories were adapted from a literature review by ideas 42 about behavioral economics approaches for PBC in Zambia. ${ }^{15}$ 


\title{
TABLE 1 PBC APPROACH GROUPINGS ${ }^{\dagger}$
}

Supervision, training, and values clarification

- Training and education ${ }^{\ddagger}$ reinforce or supplement providers’ existing knowledge and address biases.

- Managerial approaches include supportive supervision, provider detailing, mentorship, peer support, audits, monitoring, and feedback.

- Individual process improvements are tools such as job aids and reminders.

- Supplementary patient-facing materials assist providers in counseling patients.

- Values-clarification exercises help a provider become aware of any values they have that may be influencing the care they provide to clients.

\section{Regulations, policies, and updates}

- Organizational changes for systems-wide change.

- Institutional process improvements, such as checklists and case sheets.

\section{Incentives}

- Financial and non-financial incentives reward providers for desired behavior.

\section{Infrastructure, supplies, and workload}

- Infrastructure improvements to facilities.

\section{Social accountability approaches}

- Social accountability approaches involve community members or clients in dialogue with the goal of holding providers accountable.

\begin{abstract}
${ }^{\dagger}$ Although some of these approaches (particularly approaches within the category of infrastructure, supplies, and workload) are typically within the purview of service delivery partners, they can be considered PBC approaches in circumstances when they are implemented with the goal of changing provider behavior.

†Training and education solely aimed at providing clinical knowledge and skills would not be considered a PBC approach alone but could be part of an intervention designed to positively influence provider behavior.
\end{abstract}

Finally, the categories were expanded to include social accountability, which was determined to fall outside the identified groupings.

\section{Barriers addressed by intervention}

Although some of the intervention categories are best suited for addressing certain barriers rather than others-e.g., training providers to address knowledge and competency barriers or infrastructure improvements to address structural and contextual barriers - the barrier(s) addressed by each intervention were determined by holistically assessing the intervention and evaluation, i.e., by examining what, if any, rationale for the intervention was given, what approaches were used, and what outcomes were measured. 


\section{Results}

The literature search yielded a total of 18 documents including 17 evaluations of PBC interventions in seven of the nine OP countries, with 11 documents found in grey literature and seven found in published literature. Table A.1 (see Appendix $2^{f}$ ) summarizes the evaluation studies, the PBC approaches used, and the study outcomes.

\section{Geographical distribution}

The PBC evidence spanned seven of the nine OP countries; all but Mauritania and Côte d'Ivoire were represented. Five studies took place in Senegal:;6-20 four each in Guinea, ${ }^{18,21-23}$ Mali, $^{18,24-26}$ and Niger; ${ }^{18,27-29}$ three in Togo, ${ }^{18,30,31}$ and one each in Benin ${ }^{18}$ and Burkina Faso ${ }^{32}$ (Figure 2).

\section{Study design}

We found the strength of the evidence on effective PBC approaches in the OP countries to be weak. Some of the

${ }^{\mathrm{f}}$ Available at breakthroughactionandresearch.org/wp-content/ uploads/2020/03/PBC-Landscaping-FP-OP-Supp.pdf assessments used routine monitoring data without a design or analytical techniques (such as randomization, comparison groups, triangulation) that allow for greater confidence in causal inferences drawn. Of the 17 evaluations included in the landscaping, 12 (71\%) employed a non-randomized, pre-post study design, comparing endline results with baseline data or historical program data with no comparison group. ${ }^{16-19,22-28,33}$ Some of these used regularly collected monitoring data to evaluate changes in outcomes over time, ${ }^{23,25}$ while others combined monitoring data with client-provider observations or other intervention-specific methods. ${ }^{17,27,28}$ Only one study employed a randomized design, ${ }^{32}$ while two studies used a quasi-experimental design with a comparison group. ${ }^{21,29}$ Two studies employed an exclusively qualitative assessment (Figure 3). ${ }^{20,31}$

\section{Barriers addressed}

Table A.1, provided in the appendix, presents the barriers addressed by each PBC intervention. Of the 17 studies, most (13 studies) addressed structural and contextual barriers ${ }^{16,18-22,24,26-30,32}$ as well as knowledge and

\section{FIGURE 2 GEOGRAPHICAL DISTRIBUTION OF STUDIES IDENTIFIED $(\mathrm{N}=17)$}

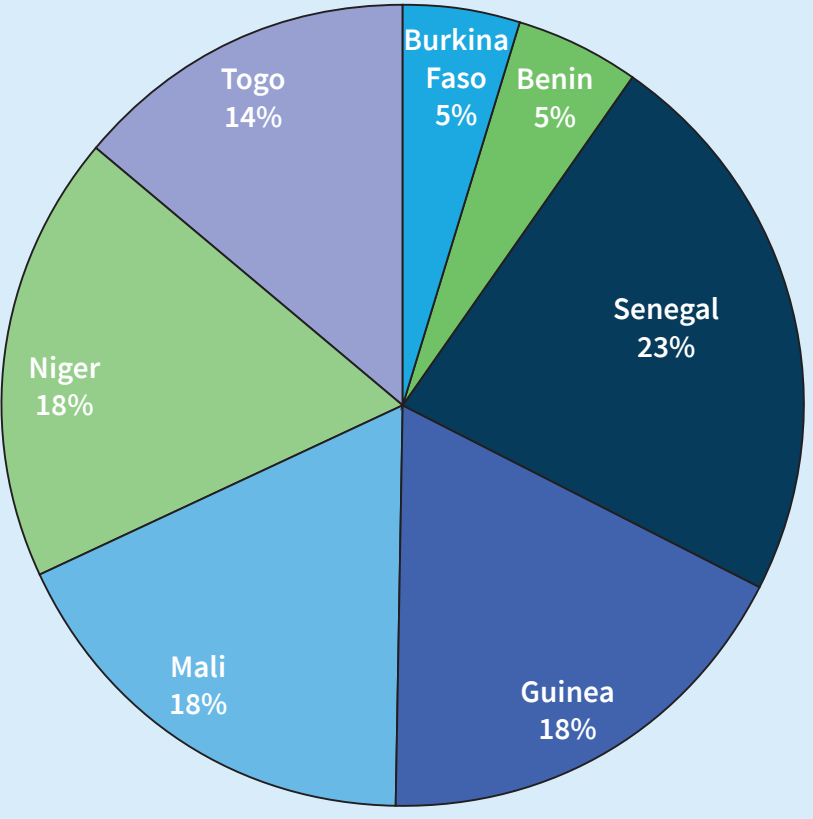

\section{FIGURE 3 STUDY DESIGN DISTRIBUTION}

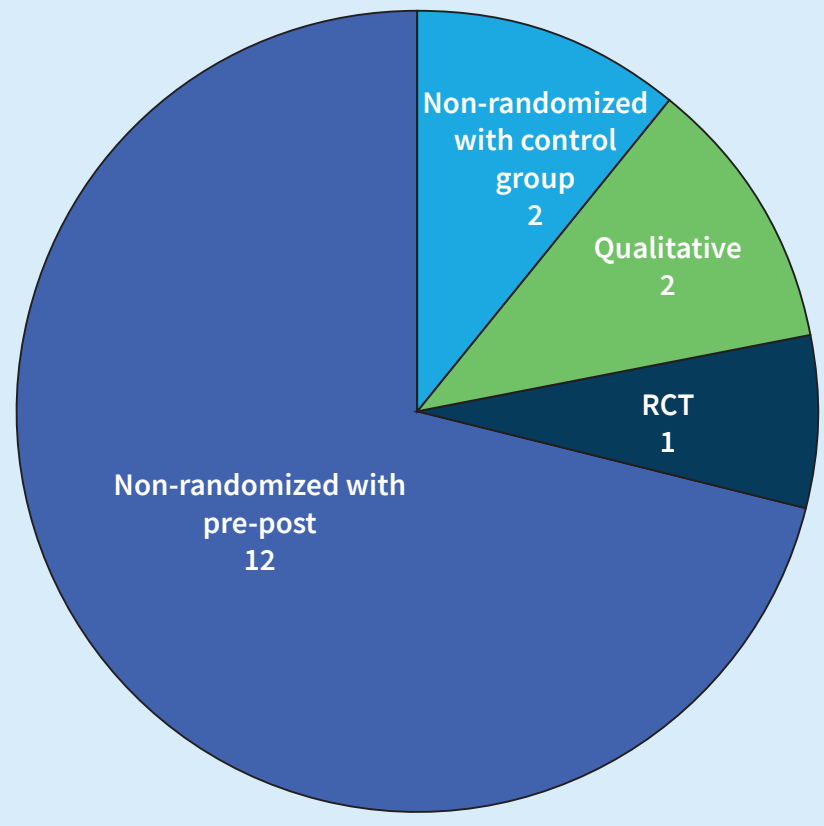


competency barriers (13 studies). ${ }^{17-19,21,23,25-32}$ Fewer than half (six studies) addressed attitudinal barriers. ${ }^{20,21,24,27,29,30}$ Supervision, training, and values clarification approaches typically addressed knowledge/competency and attitudinal barriers. Regulations, policies, and updates, as well as infrastructure, supplies, and workload approaches, most often addressed knowledge/competency or structural/ contextual barriers. Incentives addressed structural/ contextual and attitudinal barriers. Social accountability approaches, which in both cases were combined with managerial approaches, addressed structural/contextual and attitudinal barriers as well.

\section{Outcomes assessed}

Table A.1 presents each PBC evaluation and the type of outcome assessed, with significant associations (quantitative assessments) or attributed relationships (qualitative approaches) denoted by (+). None of the documents presented negative or neutral associations for their primary outcomes. The majority of studies (15 of 17) examined client-level outcomes ${ }^{16-18,20-25,27-32}$ such as: new FP adopters, couple-years of protection, ${ }^{\mathrm{g}}$ proportion of clients recalling FP messages, proportion of clients receiving counseling on FP methods, ${ }^{h}$ proportion of clients adopting a method after receiving counseling, and client satisfaction. Few studies examined provider- and facility-level outcomes (nine and seven studies, respectively), such as increased provider FP/RH knowledge and quality of services offered at the facility level.

\section{PBC approaches}

We describe findings from the literature review using the organizational categories that emerged through the screening process. While most of the studies identified (15 of 17) used more than one PBC approach, we highlight examples of interventions falling under each category.

\footnotetext{
${ }^{8}$ Couple-years of protection is the estimated protection provided by FP services during a one-year period, based upon the volume of contraceptives sold or distributed (MEASURE Evaluation Family Planning and Reproductive Health Indicators Database, accessed 7 January 2020).

${ }^{h}$ For many of these studies, especially those with facility-level interventions, not all clients always receive FP counseling. Thus, part of the PBC intervention was to increase the number of clients who were counseled on FP or to incorporate such counseling into more FP visits.
}

\section{Supervision, training, and values clarification}

The majority (16 out of 17) of PBC studies included in this landscaping aimed to address provider knowledge and clinical competency through supervision, training, and values-clarification-type approaches. These approaches include: training and education to reinforce or supplement providers' existing knowledge and address biases; managerial approaches that include supportive supervision, mentorship, peer support, audits, monitoring, and feedback; individual process improvements such as job aids and reminders; supplementary patient-facing materials that assist providers in counseling patients; and values-clarification exercises that help a provider become aware of any values they have that may be influencing the care they provide to clients.

Managerial approaches and training and education were the most commonly implemented strategies, each used by 12 studies, followed by institutional process improvements and individual process improvements and supplementary patient-facing materials, each used by four studies. Values clarification approaches were not as widely studied and were only implemented in one study. ${ }^{30}$

A number of projects adopted multiple training and supervision approaches to address knowledge and competency barriers. For example, the ACQUIRE Project in Guinea focused on addressing knowledge and competency barriers through an approach that combined training and education, managerial approaches, organizational changes, individual process improvements, and supplementary patient-facing materials. ${ }^{23}$ The intervention involved updating provider knowledge of long-acting and permanent methods (LAPMs) through training and addressed other provider behaviors via approaches such as clarifying job expectations, facilitative supervision, updating communication materials, and developing job aids. The intervention, which also included demand-side activities with clients, succeeded in increasing intrauterine device (IUD) use among clients, resulting in an increase of couple's years of protection from 130 in 2004 to 2,450 in 2006 .

One of the few studies addressing attitudinal barriers was Evidence to Action (E2A), which used a training approach in Togo. ${ }^{30}$ Post-abortion care (PAC) providers were trained in rights-based care and underwent values clarification training to address issues such as provider bias toward youth and the need to provide counseling 
and FP methods whether abortions were induced or spontaneous. Mugore et al. did not report on changes in provider attitudes across the intervention period but found that voluntary contraceptive uptake among young PAC clients increased from $0 \%$ before the intervention to $41 \%$ afterwards.

Despite the emphasis on supervision and training-based approaches to address provider behavior, few studies examined provider- and facility-level outcomes. However, a couple of programs that focused on provider attitudes and technical competence showed some promising improvements on provider outcomes. In Guinea, Bradley et al. assessed provider attitudes and their ability to solve problems after a 15-month intervention period involving a series of tools called COPE, which allows providers to identify their own problems, decide what help is needed, and seek their own solutions. ${ }^{21}$ They found that post-intervention, providers self-reported improved personal communication skills, improved diagnostic skills, improved home care instructions, improved immunization practices, and somewhat improved prescribing practices. Providers also reported that they had new knowledge and awareness of client rights and expectations and new awareness of their role in influencing change. In Senegal, Suh et al. found that formative supervision, which combines observation with a problem-solving approach to clinical, logistic, information, education, and communication issues in health service delivery, improved provider technical competence and logistics management performance. ${ }^{19}$

\section{Regulations, policies, and updates}

Nine studies included new policies, regulations, and updates to help to expand access to services and enable providers to provide more comprehensive care. In Senegal, there was an effort to decentralize care from national and regional hospitals to district health centers by implementing a new treatment protocol for PAC in the district health centers. ${ }^{15}$ The change in protocol was complemented by supervisory visits and the COPE tool, which enabled providers to identify problems and develop follow-up action plans. The study examined the cost associated with the intervention and evaluated the feasibility and impact of decentralized care for spontaneous abortion in rural areas of Senegal and found that the new model of care decreased the average cost per patient by 3,500 CFA francs, or about 6 USD. Although client satisfaction was not assessed at baseline, 94\% of clients reported being satisfied with services after the intervention.

\section{Incentives}

Only one study, a University Research Co. (URC) study in Senegal, used financial incentives as a PBC approach in the region to improve the quality of a variety of health services, including FP, maternal and newborn health, and infectious diseases. ${ }^{20}$ The results-based financing (RBF) program granted financial awards to providers and facilities if they attained quality goals. A qualitative evaluation found that the intervention improved provider behavior and quality of care. Qualitative findings also suggested that RBF helped transform the organizational culture within facilities toward more responsive care with strategic and collaborative use of resources.

\section{Infrastructure, supplies, and workload}

We identified four evaluations that studied infrastructure improvements such as integration of FP into other health facility services. ${ }^{16,21-23,26-29,32}$ Bossyns and colleagues evaluated an intervention that utilized infrastructure improvements in addition to regulations, policies, and updates as well as training and education in Niger. ${ }^{27}$ The intervention included the abolition of special FP clinics and integrated FP services into other health center activities to make consultations more responsive to patients' needs. Additionally, in order to overcome provider attitudes or biases that may act as barriers to FP access, policies were instated that mandated that providers must offer contraceptives to all women attending child health, antenatal, and postnatal care visits. Providers were also trained in interpersonal communication and were taught tips on how to engage in dialogue on contraceptives with clients. The intervention resulted in an increase in FP uptake among eligible female clients presenting at the health center from $1 \%$ to $29 \%$.

The USAID ASSIST Project in Guinea addressed structural and contextual factors through a program that introduced infrastructure improvements coupled with managerial approaches and organizational changes with the goal of increasing use of FP after PAC. The infrastructure and workload improvement component involved setting up a dedicated, private FP counseling station and division of FP tasks among providers. Additionally, a midwife was designated to monitor FP activities and provide FP counseling on the day of maternal discharge. The program measured changes in client-level outcomes, such as the proportion of women who received FP counseling as part of routine postpartum care, the proportion who left the health facility with an FP method, and the proportion of couples counseled for FP. ${ }^{25}$ The percentage of women 
receiving a FP method before leaving the clinic reportedly rose from $11 \%$ to $59 \%$ following exposure to the program. While the quality of counseling was reported to have improved significantly following the intervention, no data were presented to demonstrate this improvement.

\section{Social accountability approaches}

Social accountability approaches involve community members or clients in dialogue with the goal of holding providers accountable. We identified two studies that evaluated social accountability approaches. ${ }^{19,24}$ One study examined community-level outcomes. ${ }^{24}$ This intervention, under the Supporting Access to Family Planning and Post-Abortion Care project in Mali, built the capacity of providers and used a social accountability approach wherein providers were connected to communities for dialogue about power dynamics and the definition of quality services. This provided a venue for community members to share feedback with providers. As part of a continuous quality improvement process, community reflections expressed during these meetings were documented and incorporated into plans of action for the providers, who were monitored using routine data collection. There was a reduction in community complaints from 400 in 2016 to 47 in 2018, and the credibility of providers in the community improved. Client-level outcomes were also assessed to find that, across the intervention period, there were 57,777 new FP users, a majority of whom used LARCs.

\section{Project and actor mapping of current PBC approaches in FWA}

This review identified 22 projects utilizing PBC approaches that have been active in the FWA region within the past 5 years (Table A.2 in Appendix 2i). Case studies 1 and 2 highlight examples of current projects addressing provider behavior, including lessons learned for future implementation and evaluation. The case studies were based on informational interviews.

\section{Case Study 1}

\section{Quality Assurance Project in Niger}

Camber Collective and Animas Sutura

Rationale: Niger has the highest fertility rate globally, at 7.6 children per woman in 2012, yet both men and women report wanting more children than they currently have. ${ }^{34}$

Intervention: To better understand FP dynamics in Niger, The William \& Flora Hewlett Foundation engaged Camber Collective to provide a strategic analysis of the demand landscape. Following this analysis, Camber Collective aimed to improve the quality of the client-provider interaction by giving providers tools including counseling cards and flip charts, providing communication training, and implementing monthly supportive supervision visits to strengthen their FP counseling. ${ }^{29}$ Camber Collective developed an innovative tool to segment clients during FP consultations to identify homogenous groups that are receptive to certain counseling messages. As opposed to segmenting based on demographic characteristics such as residence or age, attitudinal segments were determined based on key variables, including past contraceptive use behaviors, proactivity, social norms, and attitudes and beliefs. Each segment has a predicted potential increase in modern contraceptive use given segment-appropriate FP counseling.

At the beginning of a counseling visit, the provider asks a client 12 questions and tallies the scores, which determines what profile the client belongs to: healthy proactives, traditional autonomists, sheltered skeptics, modern elites, or conservative passives. ${ }^{35}$ Each profile has corresponding counseling cards to aid the provider in starting a conversation about FP. For example, many healthy proactives are open to or already use modern methods of FP, and thus providers can discuss longterm modern methods with this group. Traditional autonomists often use traditional methods but have the potential to convert to modern methods, and emphasizing their autonomy is recommended. Sheltered skeptics aren't familiar with FP but may be willing to use it if they receive counseling and education from a trusted source. Modern elites are the most likely to already be using modern methods and are also the most likely to be open to a conversation around limiting once they have reached their desired family size. Lastly, conservative passives are the least interested in use of modern FP methods and
'Available at breakthroughactionandresearch.org/wp-content/ uploads/2020/03/PBC-Landscaping-FP-OP-Supp.pdf 
may believe it is a sin; therefore, counseling to these clients would likely be the most difficult and values around use of FP would need to be approached more carefully.,35 Provider training involved a coach to introduce the tool and demonstrate that it did not require any added time for counseling and allowed the provider to establish a meaningful connection with their clients quickly.

Evaluation: In collaboration with Animas Sutura, a pilot project was conducted in 36 primary health centers (12 pilot intervention, 24 comparison) in Maradi and Tillabéry, Niger. A quasi-experimental design was employed: The 12 pilot intervention sites were selected by the Ministry of Health and Animas Sutura out of 297 possible sites. The 24 comparison sites were randomly selected by Camber and Animas Sutura from a list of sites in the same health districts as the pilots. Baseline data were collected from clients at the facilities before implementation. Following implementation of the intervention, exit interviews ( $n=404$ for the intervention, $n=367$ for the comparison) were conducted with clients to assess a variety of indicators related to implementation, satisfaction, awareness, knowledge, consideration/acceptance, and use of FP. A coach provided monthly follow-up and support for health care workers to aid them in integrating the segmentation tool.

Results: Results of the pilot study indicated that pilot sites showed improvement in indicators of all six categories compared with control sites at endline. Examples of improvements in unadjusted indicators for each of the six categories include:

- Implementation: A greater percentage of women in pilot sites compared with comparison sites reported that FP sensitization sessions took place in the waiting room (93\% vs. $87 \%$ ) and in the community ( $83 \%$ vs. $78 \%)$.

- Satisfaction: The percentage of women reporting having confidence in health care workers was significantly higher in pilot sites (99\%) compared with comparison sites (95\%).

- Awareness: On average, women in pilot sites reported knowing about a greater number of FP methods (3.51) compared with those in comparison sites (3.19), and this difference was statistically significant.

IIn this study, women were coming for FP services and this group constituted a small proportion of the total number of women who received consultations.
- Knowledge: Ninety-eight percent of women interviewed at pilot sites felt they had enough information to make a good decision about FP, which was significantly higher than the $93 \%$ of respondents in comparison sites who reported having enough information.

- Consideration/acceptance: One third (33\%) of women in pilot sites reported it was acceptable for women to decide on the number of children they have, compared with $23 \%$ in comparison sites.

- Use of FP: The percentage of women currently using a modern method was $84 \%$ in the pilot and $78 \%$ in the comparison sites at the time of the exit interviews.

Key challenges: At baseline, the comparison sites had better performance on these indicators compared with the national average, so the increase in the pilot sites compared with the comparison sites was not as great as anticipated, which may have been affected by a lack of statistical power. Additionally, process evaluations demonstrated that some pilot sites scored lower than others on their consistent use of the segmentation counseling tools. The improvements across the indicators listed above were even more significant when isolating those sites where the training coach had observed a more consistent application of the counseling tool and approach.

Key lessons learned for implementation: The commitment and involvement of the Regional Directorate of Public Health as well as the districts was instrumental for successful implementation and monitoring. Utilization of a cascading approach and practical exercises in the training of providers proved to be efficient and effective in this intervention. Providing direct and constant monitoring to the field teams was also useful, especially early on in the intervention. Once providers were sufficiently familiar with the tool, they greatly appreciated how simple it was to employ and seemed to gain a small amount of intrinsic motivation to use the tool and provide a higher quality of counseling interactions, so much so that they requested that the district managers be trained on the approach so they could continue after the pilot was finished. Successful implementation and sustainability of the intervention was ensured by setting up focal points to ensure coaching at each facility, setting up quality assurance teams, and creating information-sharing systems to ensure better communication throughout the health system. 
Key lessons learned for evaluation: As demonstrated in this pilot, effective evaluations should include a control group and collect baseline and endline data from both intervention and control groups to be able to account for baseline differences between the two groups.

Additionally, because some pilot sites performed better than others, ensuring collection of process evaluation indicators is key to understanding whether the evaluation worked as it was intended.

Next steps, scale, and sustainability: The intervention showed promise in improving indicators of implementation, satisfaction, awareness, knowledge, consideration/ acceptance, and use of FP. The Ministry of Health was very interested in the results as well the low-cost implications for scaling the intervention and secured funding from the West African Health Organization to include the segmentation in provider training nationwide.

\section{Case Study 2 Beyond Bias in Burkina Faso YLabs, Camber Collective, and Pathfinder International}

Rationale: Design and test scalable innovative solutions to address provider bias toward serving youth ages 15-24 with FP services in Burkina Faso, Pakistan, and Tanzania. ${ }^{36}$

Intervention: With funding from the Bill \& Melinda Gates Foundation, the project consortium partnersPathfinder International, YLabs, BERI, and Camber Collective-designed an intervention and evaluation framework to address and measure provider bias. As part of formative work, Camber Collective conducted a literature review and a provider segmentation analysis with 811 providers. YLabs conducted qualitative primary research with 373 providers, youth, and community members across the three countries. This formative work identified and validated 11 key drivers of provider bias in Burkina Faso, Tanzania, and Pakistan. ${ }^{10}$ With this information, YLabs and Pathfinder rapidly designed, tested, and refined potential interventions in the three countries. This iterative, human-centered design approach of solution development is referred to as "prototyping." In total, 29 facilities were engaged in the prototyping process across a 3-month period, representing 103 providers and facility managers. The results have been used by
Pathfinder as the basis for a larger 12-month intervention with 227 facilities and a corresponding mixed-methods randomized controlled trial.

Using this human-centered design approach, YLabs and Pathfinder developed a three-part adaptive solution to address provider bias, which was carried out at public clinics in Burkina Faso and Tanzania and at private clinics in Pakistan. First, a one-day event (called "Summit") was held, during which providers were exposed to stories from youth about the negative impact they sustained from being denied contraceptive services. Providers were led through guided reflection exercises to support them to recognize their own biases and create an action plan with their peers to change their biased behaviors toward youth clients. YLabs and Pathfinder worked with providers to identify what was feasible, given time constraints on the providers' availability to participate in the intervention, in addition to their normal duties. The event also featured respected guest speakers such as ministry of health representatives to give providers institutional permission and encouragement to serve youth clients.

Following the one-day event, YLabs and Pathfinder initiated a multi-week ongoing peer learning forum (called "Connect") that was designed to support providers to apply unbiased practices in their day-to-day work, address technical misinformation, and provide a space for ongoing group problem-solving. The second phase built on the one-day event, using the principle of repeated reinforcement to support habit change. In Pakistan, the format of this forum was via a WhatsApp group with text, video, and audio content and a weekly curriculum with different topics of interest to providers. In Burkina Faso and Tanzania, the forum was facilitated via bi-monthly in-person meetings led by the facility managers.

Lastly, the third component of the intervention used social reinforcement and performance-based recognition for progress made as an incentive for providers to make progress in reducing biased behaviors toward youth. The incentive was non-monetary, as financial incentives proved to be less effective in early prototyping. Changes in provider bias were measured relative to the baseline as opposed to being measured in absolute terms and providers were given feedback on their progress and recommendations of focus areas for further improvement. Progress was assessed at the facility level in Burkina Faso and Tanzania, and reinforcement came from facility managers. In Pakistan, because the intervention took place at private facilities with individual providers, progress was 
measured at the provider level, which was functionally equivalent to the facility-level.

Evaluation: To assess the success of this prototype in reducing provider bias, a quantitative exit interview was implemented among youth about their experiences seeking care at the facility. Pre-post mystery client visits were also used to assess the client-provider interaction. Additionally, qualitative findings on the implementation of the interventions were collected from providers, youth, and facility managers.

Results: Results of the prototyping were preliminary, qualitative, and process-oriented in nature, as the primary purpose was to inform the scale-up of the intervention for future testing in a larger study group of 227 facilities with a randomized controlled trial. However, the results showed that the intervention was feasible in these three settings and was promising in reducing providers' attitudinal and behavioral bias toward unmarried youth. The Connect WhatsApp forum built a professional community of private sector providers in Pakistan, with an average of 109 messages sent per week by providers. In Burkina Faso, the in-person Connect discussions were popular among both providers and facility managers. Incorporating other relevant content, such as business and marketing skills for private providers in Pakistan, was useful for gaining provider buy-in and sustained engagement over time. Through prototyping the non-financial recognition program, the team found that providers' motivation to improve services increased as a result of the offer of social recognition. This was assessed through qualitative interviews and pre-post surveys with providers. In particular, Pathfinder and YLabs learned that rewarding facilities based on their relative progress in reducing bias in services was more motivating than ranking clinics against each other.

Key challenges: In Burkina Faso, a high social desirability bias among providers hindered providers' recognition of and willingness to publicly admit their own biases. Multiple iterations of the intervention in the prototyping phase were required to effectively enable providers to admit bias and commit to action on it. Formative prototyping work identified challenges in measuring bias from the perspective of clients, including lack of a clear, measurable definition of bias, clients' reluctance to provide feedback about their service experience, and youth clients' tendency to rate services as high-quality even if service was denied or improper/biased counseling was given.
Key lessons learned for implementation: The results from Beyond Bias's prototyping phase strongly suggest that an integrated behavior change strategy to address provider bias is crucial. Specifically, this means moving beyond one-time training and incorporating light-touch sustained reinforcement techniques to shift professional norms in the providers' workplace. Encouraging empathy among providers toward youth is key to reducing bias in the client-provider interaction but must be paired with structured reflection and clear, measurable targets with the promise of social recognition for providers to commit to action. For example, videos of girls and young women discussing FP were played to build empathy among providers. It was important to acknowledge that bias occurred without shaming providers in order for providers to openly admit their bias and commit to action on it. Additionally, it was important to understand providers' practical motivation and needs, such as business sustainability in the case of the Pakistani providers, and then add in content to the intervention that providers perceived as directly relevant to their day-to-day concerns.

Finally, segmentation of providers into different behavioral subgroups was useful to design a more targeted intervention that was tailored to the diverse motivational profiles of providers. The use of the human-centered design process enabled YLabs and Pathfinder to explore and test a wide range of intervention concepts directly with providers and youth and evolve the concepts collaboratively through multiple cycles of rapid testing. The combination of the two methods-segmentation and human-centered design-allowed the project consortium to create an intervention that was feasible to implement and desirable to providers and youth as well as having promising impact for behavior change based on the preliminary findings from prototyping.

Key lessons learned for evaluation: Exit interviews with young people were not always sufficient in measuring bias, as young people can be reluctant to provide feedback about bias and may not recognize what biased care looks like. Exit survey instruments should use questions that are focused on the provider's objective behavior rather than on how "satisfied" the client was or on other emotions (feeling "welcome," "happy," etc.). In addition, the best-performing survey questions used dichotomous ("yes/no") answer options rather than multi-point scales. Employing mystery clients was an effective way to complement the exit survey interviews. 
In response to a gap in the evaluation literature, the Beyond Bias project developed a new framework for assessment of bias called the "Six Principles of Unbiased Care," which was developed in partnership with WHO advisors to the project. Each of the six principles has measurable indicators and corresponding client survey questions.

Next steps: The intervention is being implemented and evaluated with a randomized controlled trial by Pathfinder International in 227 facilities across the three countries, with an intended end date of September 2020. 


\section{Summary of Findings and Recommendations}

Despite many gaps in the documented evidence, there are many ongoing PBC interventions in FWA that appear promising regarding their expected impact on provider behaviors and client outcomes. This landscaping review examined 17 evaluations of interventions to change provider behavior related to FP services in this setting. We present a summary of key findings with recommendations for future programs and research on PBC interventions below.

\section{Key findings}

\section{Geographic distribution}

- We identified evaluations of PBC approaches in seven of the nine OP countries, with the exception of Mauritania and Côte d'Ivoire. Most evaluations focused on interventions in four countries: Senegal, Guinea, Mali, and Niger, the four countries with the lowest unmet need for FP.

\section{Research and study design}

- Many of the evaluations relied on relatively weak study designs, with most studies not including a comparison group. Many also collected routine monitoring data without design or analytical techniques to allow causal inference.

- Most studies included in the review assessed multiple interventions, often including both supply-and demand-side components, and did not attempt to parse out the effects of each of these components.

\section{Barriers addressed}

- Most of the PBC interventions evaluated so far have addressed knowledge and competency and structural and contextual barriers through managerial approaches, training and education, and institutional process improvements. There is limited evidence on approaches used to address provider attitudes and provider bias, a barrier that SBC programming is well-positioned to address.
- Among the limited number of studies that have assessed provider bias, findings indicate that bias is difficult to measure, and some sensitivity is required to ensure that bias is acknowledged without blaming providers and that providers feel supported and have a stake in the intervention.

\section{Outcomes assessed}

- The outcomes assessed in the documented evaluations were mostly limited to client-level outcomes such as FP uptake, without assessing changes in provider knowledge and competencies.

- Furthermore, quality of care was not assessed in most of the reviewed evaluations. The few studies that examined quality of services examined provider perspectives only.

- The evaluations found positive effects of PBC interventions on primary outcomes.

\section{PBC approaches}

- Provider-driven approaches, such as the COPE method described by Bradley and Cisse, may positively affect the organizational culture of health facilities and systems and help create an enabling environment for sustained behavior change.

- There is limited evidence on social accountability and incentive-based approaches as well as approaches that address policy and regulatory-related issues.

\section{Programmatic documentation}

- Pathways for how the proposed PBC interventions achieved the intended objective were not always explicitly shared, making it difficult to determine how the PBC intervention influenced the intended outcomes-particularly when more distal outcomes were reported.

- A limited number of programs have documented and shared formative research used to inform the intervention design. 


\section{Vulnerable populations}

- Several projects focused on LAPM, postpartum FP, and PAC, but there appeared to be less evidence on approaches aimed at addressing issues related to provider bias toward adolescents and nulliparous women despite evidence on this recognized barrier.

\section{Costs and cost-effectiveness}

- There is limited evidence on the costs and cost-effectiveness of PBC approaches. Only one study examined the cost of the intervention, and none of the included studies examined cost-effectiveness.

\section{Recommendations}

\section{Geographic distribution}

- More evidence needs to be generated in FWA to determine which approaches work best to address barriers to provider FP behavior in certain contexts. Specific countries with a small body of evidence include Benin, Burkina Faso, Côte d'Ivoire, and Mauritania, which, in addition to Togo, are the OP countries with the highest unmet need for FP.

\section{Research and study design}

- There is a need for more rigorous evidence on what PBC approaches work best in OP countries. To increase the confidence in conclusions about what works in PBC, approaches using randomized designs, comparison groups, and/or triangulation with routine or program monitoring data should be considered when feasible.

- Where possible, behavioral measures and health outcomes should be captured in addition to more intermediate factors, such as changes in knowledge, attitudes, and beliefs.

- Future programs should consider evaluation designs that are able to assess the relative contribution of elements when PBC interventions include a package of approaches and assess which approaches are most effective when combined.

- The costs of interventions should be captured and, when there is rigorous effectiveness data available, the cost-effectiveness of interventions should be assessed.

\section{Barriers addressed}

- To address context-specific barriers to service provision, formative research such as structured diagnostic processes should be used to identify determinants of provider behavior.

- PBC programs should conduct and document formative work to understand the social and gender norms that influence provider behavior. More evidence is needed to understand whether normative approaches, such as values clarification, can improve the quality of providers' interpersonal communication with clients.

- In addition to addressing knowledge and competency and structural and contextual barriers, interventions should aim to address attitudinal barriers including provider bias using SBC approaches.

- Programs should be sensitive in designing approaches that address provider bias to ensure that providers feel supported and empowered rather than feeling blamed for holding biases.

\section{Outcomes assessed}

- While the goal of PBC interventions is ultimately to improve outcomes for clients, more evidence is needed on the intermediate results: changes to providers' knowledge, attitudes, and behaviors. Evaluations of PBC interventions should be sure to collect both provider-level and client-level outcomes to help elucidate how changing provider behavior is linked with improved client outcomes. It would also be useful to understand the strength of the relationship between PBC interventions and intermediate outcomes and FP utilization.

- Further research is needed to understand whether improving the behaviors/practices of health providers influences the quality of care provided and to identify the most effective SBC approaches to improve quality of care. $\mathrm{PBC}$ program implementers and evaluators should assess changes in quality of care from multiple perspectives.

\section{PBC approaches}

- Donors should consider further testing of social accountability and incentive-based approaches to better understand the range of interventions that can effectively be deployed to improve provider behaviors. 
- SBC implementing partners should apply approaches that empower providers to identify problems and create solutions in varying settings to see whether improvements in organizational culture occur across settings.

\section{Programmatic documentation}

- To better understand what makes PBC interventions work and to what extent they achieve the desired outcome, there is a need for programs to develop and monitor program theories of change.

- Programs should comprehensively document and share evidence generated through formative research and during the design and development of PBC approaches to inform future activities.

- Programs should share lessons learned so that the evidence base can provide a more robust understanding of what PBC approaches work and do not work in different settings and with different types of providers.

\section{Vulnerable populations}

- Because provider bias has been found against youth and nulliparous women in FWA, evaluations of programs addressing provider bias should collect disaggregated data to allow for subgroup analysis.

\section{Costs and cost-effectiveness}

- Future evaluations should collect cost data to determine the cost-effectiveness of PBC approaches. 


\section{References}

1. Le Partenariat de Ouagadougou. 2018. Map of projects. (Accessed 2 October 2019, at https://map.partenariatouaga. org/.)

2. United Nations Department of Economic and Social Affairs, Population Division. 2019. "Estimates and projections of family planning indicators 2019." New York: United Nations.

3. Jain, AK, J Townsend, and S RamaRao. 2018. "Proposed metrics to measure quality: an overview," Working Paper Three of the Measuring and Monitoring Quality of Care Project. New York: Population Council.

4. Tumlinson, K. 2016. "Measuring quality of care: a review of previously used methodologies and indicators," Working Paper Two of the Measuring and Monitoring Quality of Services and Quality of Care Project. New York: Population Council.

5. Manning, A, M Schaaf, and Respectful Maternity Care Council. 2018. "Disrespect and abuse in childbirth and respectful maternity care." New York: Averting Maternal Death and Disability (AMDD) Program, Columbia University.

6. Breakthrough RESEARCH. 2019. "Advancing provider behavior change programming," Research and Learning Agenda. Washington, DC: Population Council; 2019.

7. The Health Communication Capacity Collaborative (HC3). 2016. "Factors impacting the effectiveness of health care worker behavior change: a literature review." Baltimore, MD: Johns Hopkins Center for Communication Programs.

8. Transform/Phare. Côte d'Ivoire: Analyse des obstacles/ barrieres a l'offre de services de planification familiale par les prestataires, suivie d'une etude de la deviance positive. Rapport de recherche. 2017.

9. Sidze, EM et al. 2014. "Young women's access to and use of contraceptives: the role of providers' restrictions in urban Senegal," International Perspectives on Sexual and Reproductive Health 40: 176-83. doi: 10.1363/4017614

10. Camber Collective. 2018. "Beyond Bias: provider survey and segmentation findings." Washington, DC: Pathfinder International.

11. Sherard, D, S May, E Monteforte, and H Hancock. 2013. Provider Behavior Change Implementation Kit. Baltimore, MD: Johns Hopkins University.

12. Rowe, AK et al. 2018. "Effectiveness of strategies to improve health-care provider practices in low-income and middle-income countries: a systematic review," The Lancet Global Health 6: e1163-e75. doi: 10.1016/S2214-109X(18)30398-X

13. Solo, J, Festin M. 2019. "Provider bias in family planning services: a review of its meaning and manifestations," Global Health: Science and Practice 7(3): 371-385. 10.9745/ GHSP-D-19-00130

14. Tavrow, P. 2010. "Promote or discourage: how providers can influence service use." In: Malarcher S, ed. Social Determinants of Sexual and Reproductive Health: informing future research and programme implementation. Geneva: World Health Organization.
15. Zimmerman, E, V Caetano, R Banay, and J Smith. Forthcoming. "Evidence review and analysis of provider behavior change opportunities." Breakthrough RESEARCH Literature Review. Washington, DC: Population Council.

16. Cisse, CT, A Diagne, and EHO Faye. 2004. "Amélioration de la qualité des soins après avortement (SAA) en zone rurale au Sénégal," Cahiers Santé 14: 245-50.

17. Gueye, B et al. "Mentoring, task sharing, and community outreach through the tutoratplus approach: increasing use of long-acting reversible contraceptives in Senegal," Global Health: Science and Practice 4: S33-S43. doi: 10.9745/ GHSP-D-15-00328

18. Le Fonds Français Muskoka. 2016. Synthesis Annual Report 2016. The French Muskoka Fund.

19. Suh, S, P Moreira, M Ly. 2007. "Improving quality of reproductive health care in Senegal through formative supervision: results from four districts," Human Resources for Health 5: 26. doi: 10.1186/1478-4491-5-26

20. University Research Co., LLC. 2017. "Results-based financing in Senegal: opening the black box: provider response to rbf incentives for quality in Senegal," Policy Brief. Chevy Chase, MD: University Research Co., LLC.

21. Bradley, J et al. 2002. "COPE for child health in Kenya and Guinea: an analysis of service quality." New York: EngenderHealth. https://www.engenderhealth.org/wp-content/uploads/imports/files/pubs/qi/cope-child-health-report-final. pdf

22. Kaba, K et al. 2012. Championing Performance Standards to Increase Family Planning Use by Women after Postabortion Care at a Guinea Clinic. 2012; USAID ASSIST PROJECT.

23. The ACQUIRE Project. 2008. "Increasing awareness and use of long-acting and permanent contraceptive methods in guinea: case study of a pilot IUD intervention." New York: The ACQUIRE Project/EngenderHealth.

24. Diarra, S, G Alinsato, and J Nzau. 2019. "Surmonter les dynamiques de pouvoir entre petatairs de sante et client(e)s. Presentation. Care.

25. USAID ASSIST Mali. 2016. "Synthèse de la mise en œuvre de la planification familiale du post-partum au Mali. Rapport Technique." Bethesda, MD: University Research Co., LLC.

26. University Research Co., LLC (URC). 2012. "Applying quality improvement to integrate family planning in maternal health and HIV services." USAID Health Care Improvement Project. Bethesda, MD: URC.

27. Bossyns P, Miyé H, vLerberghe W. 2002. "Supply-level measures to increase uptake of family planning services in Niger: the effectiveness of improving responsiveness," Tropical Medicine \& International Health 7: 383-90. doi: 10.1046/j.1365 3156.2002.00865.x

28. Boucar, M, D Sabou, Z Saley, and K Hill. 2016. "Using collaborative improvement to enhance postpartum family planning in Niger," Technical Report, USAID ASSIST Project. Bethesda, MD: University Research Co., LLC (URC). 
29. Camber Collective and Animas-Sutura. 2016. Quality assurance pilot evaluation. Camber Collective.

30. Mugore, S. 2019. "Exploring barriers: how to overcome roadblocks impeding the provision of postabortion care to young people in Togo," Global Health: Science and Practice 7: S342S9. doi: 10.9745/GHSP-D-18-00437

31. Stevanovic-Fenn, $N$ et al. 2019. "Engaging men for effective family planning through couple communication: an assessment of two MCSP couple communication approaches in Togo." Breakthrough Research. Washington, DC: Population Council.

32. Tran, NT et al. 2019. "Post-partum family planning in Burkina Faso (Yam Daabo): a two group, multi-intervention, single-blinded, cluster-randomised controlled trial," The Lancet Global Health 7: e1109-e17. doi: 10.1016/S2214109X(19)30202-5

33. Mugore, S et al. 2016. "Improving the quality of postabortion care services in Togo increased uptake of contraception," Global Health: Science and Practice 4: 495-505. doi: 10.9745/ GHSP-D-16-00212

34. Institut National de la Statistique (INS) and ICF International. 2013. "Enquête Démographique et de Santé et à Indicateurs Multiples du Niger 2012." Calverton, Maryland, USA: INS and ICF International.

35. Camber Collective. 2015. "Increasing contraceptive use in Niger," Final Report. Camber Collective.

36. Starling, S, S Burgess, N Bennette, and H Neighbor. 2017. "Beyond Bias: literature review and expert interviews on provider bias in the provision of youth contraceptive services: research summary and synthesis." Washington, DC: Pathfinder International. 


\section{Population Council}

4301 Connecticut Ave., NW | Suite 280

Washington, DC 20008

+12022379400

breakthroughactionandresearch.org 